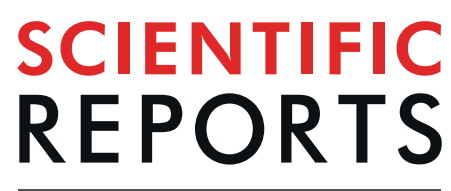

natureresearch

\title{
Genome-wide CRISPR Screen to Identify Genes that Suppress Transformation in the Presence of Endogenous Kras ${ }^{\mathrm{G} 12 \mathrm{D}}$
}

\author{
Jianguo Huang ${ }^{1}$, Mark Chen $\mathbb{1}^{2,3}$, Eric S. Xu ${ }^{1}{ }^{1}$, Lixia Luo ${ }^{1}$, Yan Ma ${ }^{1}$, Wesley Huang ${ }^{1}$, \\ Warren Floyd ${ }^{2,3}$, Tyler S. Klann ${ }^{4,5}$, So Young Kim ${ }^{6}$, Charles A. Gersbach $\mathbb{1}^{4,5}$, Diana M. Cardona ${ }^{7}$ \\ \& David G. Kirsch $\mathbb{1}^{1,2^{*}}$
}

Cooperating gene mutations are typically required to transform normal cells enabling growth in soft agar or in immunodeficient mice. For example, mutations in Kras and transformation-related protein 53 (Trp53) are known to transform a variety of mesenchymal and epithelial cells in vitro and in vivo. Identifying other genes that can cooperate with oncogenic Kras and substitute for Trp53 mutation has the potential to lead to new insights into mechanisms of carcinogenesis. Here, we applied a genomewide CRISPR/Cas9 knockout screen in $\mathrm{Kras}^{\mathrm{G12D}}$ immortalized mouse embryonic fibroblasts (MEFs) to search for genes that when mutated cooperate with oncogenic Kras to induce transformation. We also tested if mutation of the identified candidate genes could cooperate with $\mathrm{Kras}^{\mathrm{G12D}}$ to generate primary sarcomas in mice. In addition to identifying the well-known tumor suppressor cyclin dependent kinase inhibitor 2A ( $C d k n 2 a)$, whose alternative reading frame product p19 activates Trp53, we also identified other putative tumor suppressors, such as F-box/WD repeat-containing protein 7 (Fbxw7) and solute carrier family 9 member 3 (SIC9a3). Remarkably, the TCGA database indicates that both FBXW7 and $S L C 9 A 3$ are commonly co-mutated with KRAS in human cancers. However, we found that only mutation of Trp53 or Cdkn2a, but not $F b \times w 7$ or Slc9a3 can cooperate with $K$ ras $^{G 12 \mathrm{D}}$ to generate primary sarcomas in mice. These results show that mutations in oncogenic Kras and either Fbxw7 or Slc9a3 are sufficient for transformation in vitro, but not for in vivo sarcomagenesis.

Cancers frequently arise when normal cells accumulate multiple gene mutations that results in transformation ${ }^{1}$. Oncogenic mutation of KRAS is identified in several cancer types ${ }^{2-4}$, including soft tissue sarcomas (STSs) ${ }^{5}$. KRAS-mutant tumors are heterogeneous in part because of co-mutation of other genes ${ }^{6}$. Therefore, understanding the pattern of genes co-mutated with Kras may provide novel insights into KRAS-mutant cancers with clinical implications ${ }^{6,7}$. Conditional activation of an endogenous Kras $^{\mathrm{G} 12 \mathrm{D}}$ allele in mouse embryonic fibroblasts (MEFs) is sufficient to induce indefinite proliferation in vitro (immortalization), but further genetic alteration, such as mutation of $\operatorname{Tr} p 53$, is required for full transformation to enable growth in soft agar or in nude mice ${ }^{8}$. $\operatorname{Tr} p 53$ serves as a transcriptional activator that regulates multiple genes ${ }^{9}$. However, the majority of $\operatorname{Tr} p 53$ canonical target genes are dispensable for its potent tumor suppression function ${ }^{10}$. Therefore, identification of critical downstream genes that can substitute for Trp53 mutation and cooperate with oncogenic Kras to drive transformation can potentially lead to new insights into mechanisms of carcinogenesis, such as pathways by which $\operatorname{Trp} 53$ suppresses cancer. The CRISPR/Cas9 system combines Cas9 nuclease activity with targeted single guide RNAs (sgRNAs) to achieve efficient genome editing at precise sites within DNA. Cas9 targeted to a coding gene creates double strand breaks that

\footnotetext{
${ }^{1}$ Department of Radiation Oncology, Duke University Medical Center, Durham, North Carolina, 27708, USA. ${ }^{2}$ Department of Pharmacology and Cancer Biology, Duke University Medical Center, Durham, North Carolina, 27708, USA. ${ }^{3}$ Medical Scientist Training Program, Duke University Medical Center, Durham, North Carolina, 27708, USA. ${ }^{4}$ Department of Biomedical Engineering, Duke University, Durham, North Carolina, 27708, USA. ${ }^{5}$ Duke Center for Genomic and Computational Biology, Duke University, Durham, North Carolina, 27708, USA. ${ }^{6}$ Department of Molecular Genetics and Microbiology, Duke University, Durham, North Carolina, 27708, USA. ${ }^{7}$ Department of Pathology, Duke University, Durham, North Carolina, 27708, USA. *email: david.kirsch@duke.edu
} 
A

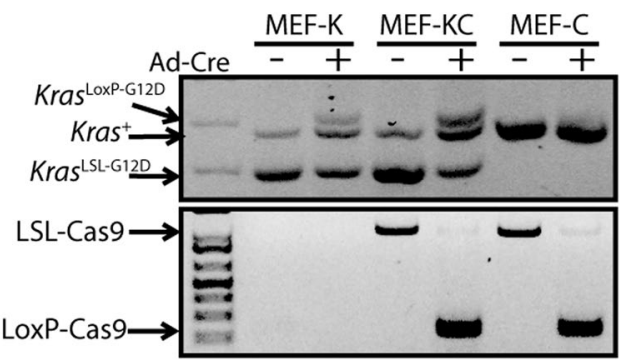

B

More than 10 passages

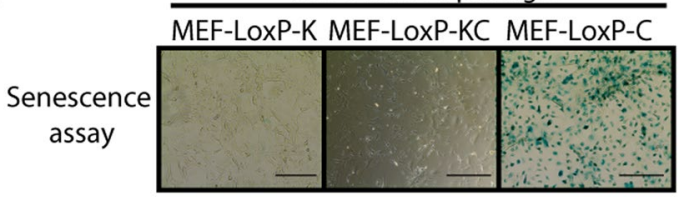

C

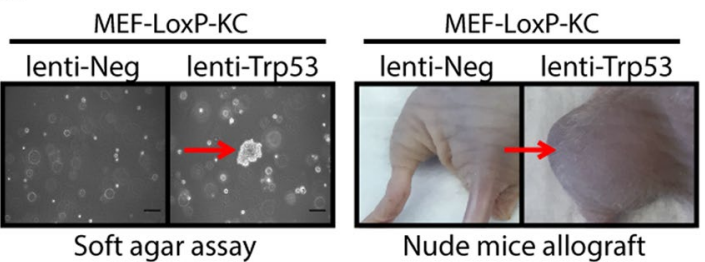

Figure 1. Immortalized MEFs expressing endogenous $\mathrm{Kras}^{\mathrm{G} 12 \mathrm{D}}$ are transformed by mutation of $\operatorname{Trp} 53$. (A) Kras $^{\mathrm{LSL}-\mathrm{G} 12 \mathrm{D}}$ MEFs (MEF-K), Kras ${ }^{\mathrm{LSL}-\mathrm{G} 12 \mathrm{D}} ;$ Rosa26 $6^{\mathrm{LSL}-\mathrm{Cas} 9-\mathrm{EGFP} /+}$ MEFs (MEF-KC), Rosa26 $6^{\mathrm{LSL}-\mathrm{Cas} 9-\mathrm{EGFP} /+}$ MEFs (MEF-C) were infected with adenovirus expressing Cre (Ad-Cre) and then genotyped using PCR to confirm recombination of the floxed STOP cassette (LoxP). (B) Ad-Cre infected MEFs (MEF-LoxP-K, MEF-LoxP-KC, MEF-LoxP-C) were cultured for more than 10 passages and were stained with reagents for the $\beta$-Galactosidase senescence assay. (C) Immortalized MEF-LoxP-KC cells were transduced with lentivirus expressing either negative control sgRNA (lenti-Neg) or a sgRNA targeting Trp53 (lenti-Trp53), and then seeded in soft agar or allografted in nude mice. MEF-LoxP-KC cells infected with Trp53 sgRNA resulted in anchorageindependent growth in the soft agar assay and tumor formation in nude mice allografts $(n=2$ or 3$)$. The result is representative of at least two independent experiments. Scale bars $=100 \mu \mathrm{m}$.

are repaired by non-homologous end-joining, which can introduce frameshift insertions and deletions (indels) that can result in loss-of-function (LOF) mutations ${ }^{11}$. Unbiased genome-wide CRISPR/Cas9 knockout screens have been applied to identify driver genes in different types of cancers, such as lymphoma ${ }^{12}$, liver tumors ${ }^{13}$, and breast cancer ${ }^{14}$, but to our knowledge have not been performed to seek genes that cooperate with oncogenic Kras for transformation.

Here, we performed an unbiased genome-wide CRISPR/Cas9 knockout screen in Kras $^{\mathrm{G} 12 \mathrm{D}}$ immortalized MEFs to search for genes that can cooperate with oncogenic Kras to drive growth in soft agar and in nude mice. From this screen, we identified several candidate genes whose mutation results in transformation. In addition, we further tested whether mutating these genes together with $\mathrm{Kras}^{\mathrm{G} 12 \mathrm{D}}$ could drive sarcomagenesis in vivo using our established CRISPR/Cas9 in vivo editing method ${ }^{15}$. Although mutating some of these candidate genes led to transformation in vitro and tumor formation when cells were injected into nude mice, mutating these genes together with ras $^{\mathrm{G} 12 \mathrm{D}}$ failed to drive sarcomagenesis in vivo. This suggests that in vitro transformation screens may fail to fully capture all of the required elements for in vivo tumorigenesis ${ }^{15}$.

\section{Results}

Mutation of Trp53 by CRISPR/Cas 9 transforms Kras $^{\mathrm{G} 12 \mathrm{D}}$ immortalized MEFs. To confirm MEFs can be immortalized by endogenous expression of $\mathrm{Kras}^{\mathrm{G} 12 \mathrm{D}}$, we isolated MEFs from $\mathrm{Kras}^{\mathrm{LSL}-\mathrm{G} 12 \mathrm{D} /+}$ mice (MEF$\mathrm{K})$, Rosa26 $6^{\mathrm{LSL}-\mathrm{Cas} 9-\mathrm{EGFP} /+}$ mice (MEF-C), as well as Kras ${ }^{\mathrm{LSL}-\mathrm{G} 12 \mathrm{D} /+} ;$ Rosa26 $^{\mathrm{LSL}-\mathrm{Cas} 9-\mathrm{EGFP} /+}$ mice (MEF-KC). We then infected the MEFs with adenovirus to deliver Cre recombinase and activate either Kras ${ }^{\mathrm{G} 12 \mathrm{D}}$ in MEF-K (MEFLoxP-K), Cas9 in MEF-C (MEF-LoxP-C) or both Kras ${ }^{\mathrm{G} 12 \mathrm{D}}$ and Cas9 in MEF-KC (MEF-LoxP-KC), as shown in Fig. 1A. The cells were cultured for greater than 10 passages and MEF-LoxP-K, MEF-LoxP-C, and MEF-LoxP-KC cells were stained for $\propto$-Galactosidase (SA- $\propto$-Gal), a biomarker for cellular senescence. SA- $\propto$-Gal activity was only significantly increased in the MEF-LoxP-C cells, indicating that only MEF-LoxP-C cells underwent senescence at late passage (Fig. 1B). In contrast, cells expressing oncogenic Kras (MEF-LoxP-K or MEF-LoxP-KC) were immortalized and did not stain for SA- $\propto$-Gal. Furthermore, transduction of early passage MEF-LoxP-KC cells with a lentivirus delivering a sgRNA targeting $\operatorname{Tr} p 53$, but not a negative control sgRNA, induced anchorage independent growth in soft agar and formed tumors when the transduced cells were allografted into nude mice (Fig. 1C). Thus, mutation of Trp53 in MEF-LoxP-KC cells was sufficient for transformation. These results suggest 
A

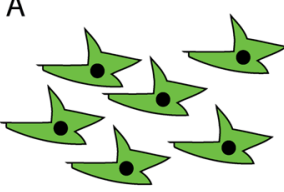

MEF-LoxP-KC

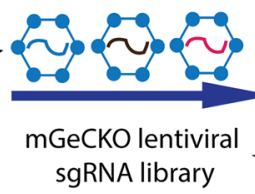
sgRNA library

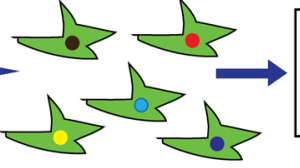

2 pools of MEF-LoxP-KC with single mutations (200 copies per sgRNA)

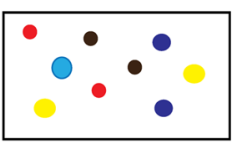

Soft agar assay (in vitro screen)

B
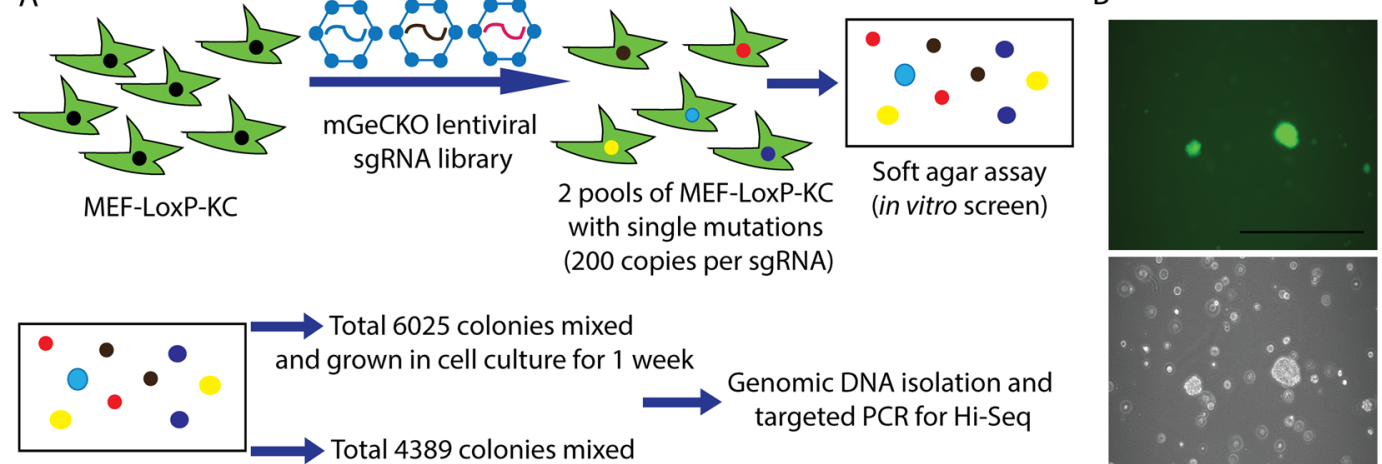

Soft agar assay

(in vitro screen)

\begin{tabular}{|c|c|c|c|c|c|c|}
\hline sgRNA library ID & Gene & \begin{tabular}{|c|} 
Rep-1 \\
before screen
\end{tabular} & \begin{tabular}{|c|} 
Rep-2 \\
before screen
\end{tabular} & $\begin{array}{c}\text { Rep-1 } \\
\text { mixed colonies }\end{array}$ & $\begin{array}{c}\text { Rep-2 } \\
\text { smixed colonies }\end{array}$ & $\begin{array}{c}\text { Ranking (Rep-1+2 } \\
\text { colonies) }\end{array}$ \\
\hline MGLibB_56014 & $\operatorname{Trp53}$ & 22941 & 21933 & 3554257 & 6045562 & 1 \\
\hline MGLibA_56035 & $\operatorname{Trp53}$ & 43586 & 67939 & 1095758 & 4167277 & 2 \\
\hline MGLibA_56034 & $\operatorname{Trp53}$ & 19276 & 65995 & 1594399 & 636149 & 3 \\
\hline MGLibA_26623 & Izumo2 & 3562 & 615 & 903731 & 2 & 4 \\
\hline MGLibB_56015 & $\operatorname{Trp} 53$ & 3825 & 1057 & 144850 & 129758 & 5 \\
\hline MGLibB_26760 & Kans/2 & 3648 & 0 & 147584 & 5 & 6 \\
\hline MGLibA_56033 & $\operatorname{Trp} 53$ & 3482 & 7719 & 50136 & 92912 & 7 \\
\hline MGLibB_56013 & Trp53 & 4135 & 953 & 67170 & 45152 & 8 \\
\hline MGLibB_09610 & $C d k n 2 a\left(p 19^{A r f}\right)$ & 7713 & 1771 & 4677 & 39 & 9 \\
\hline MGLibA_49906 & SImap & 2 & 192 & 7 & 25 & 10 \\
\hline MGLibB_55757 & Trim31 & 153 & 377 & 12 & 10 & 11 \\
\hline MGLibA_35942 & Olfr119 & 66 & 221 & 1 & 17 & 12 \\
\hline MGLibB_17100 & Fam 120c & 92 & 706 & 5 & 10 & 13 \\
\hline ....... & ....... & & & ....... & ....... & ....... \\
\hline
\end{tabular}

Figure 2. Screen with unbiased genome-wide CRISPR knockout library in MEF-LoxP-KC cells. (A) Schematic of the first unbiased genome-wide CRISPR knockout. (B) Representative colonies were identified in soft agar 3 weeks after transduced MEFs were seeded in soft agar. (C) The list of sgRNAs which were identified in colonies from the soft agar screen.

that MEF-LoxP-KC cells represent a platform for a robust genome-wide CRISPR/Cas9 knockout screen to identify candidate genes whose mutations cooperate with oncogenic Kras to result in transformation.

Genome-wide CRISPR/Cas9 knockout screen identifies Trp53 as a dominant tumor suppressor. As shown in the schematic in Fig. 2A, low passage MEF-LoxP-KC cells were infected with a lentivirus expressing mouse genome-scale CRISPR/Cas9 knockout (GeCKO) sgRNA library at 0.2-0.4 multiplicity of infection (MOI). The library contains 103,209 sgRNAs targeting 20,611 mouse genes (six sgRNAs per coding gene and four sgRNAs per microRNA) ${ }^{16,17}$. Seven days after lentiviral transduction and puromycin selection, two independent mixed pools of stably transduced MEFs with approximately 200 copies per sgRNA were plated in soft agar to positively select transformed cells by anchorage-independent growth. Colonies were detected in soft agar after 3 weeks (Fig. 2B). 6025 colonies were counted in the first group and 4389 colonies were counted in the second group. Because of the large number of colonies that grew in the soft agar screen, for each screen we combined the colonies into a single pool and recovered cells following one additional week in culture. sgRNA cassettes were amplified from genomic DNA extracted from transduced MEFs before growth in soft agar and also from MEFs recovered after the soft agar screen. Then, amplicons of sgRNA cassettes were quantified by next-generation sequencing to identify sgRNAs. Both independent screens identified all 6 sgRNAs targeting Trp53 (Fig. 2C). While Trp53 sgRNAs were dominant in both soft agar screens, we also identified several other sgRNAs targeting Izumo2, Kansl2, Cdkn2a, Slmap, Trim31, Olfr119, and Fam120c in both screens. However, when MEF-LoxP-KC cells were transduced with lentiviruses expressing individual sgRNAs to Izumo2, or Kansl2, transduced cells did not consistently yield colonies in soft agar, which suggested that inactivation of each candidate gene alone is not sufficient to cooperate with oncogenic Kras to cause transformation (Fig. S3). Instead, sgRNAs targeting these candidate genes in the screen might be passenger sgRNAs identified in transformed MEFs which had also been targeted by a sgRNA to Trp53. Furthermore, this initial screen suggested that Trp53 sgRNAs conferred a strong growth advantage during the one week expansion in culture prior to plating in soft agar, resulting in decreased representation of other sgRNAs and reduced sensitivity to identify less abundant and/or less potent sgRNAs from the soft agar colony screen. 
A

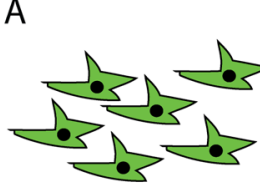

Immortalized LoxP-KC (M7a-I)

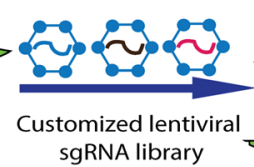
sgRNA library

2 pools

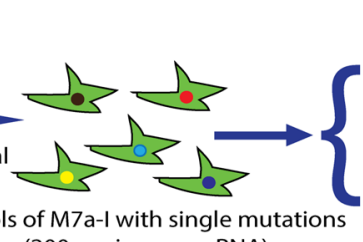

( 200 copies per sgRNA)

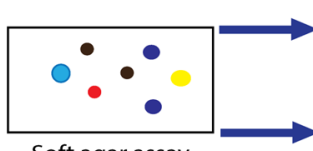

511 colonies: individually grow in cell culture for 1 week

Soft agar assay (in vitro screen)

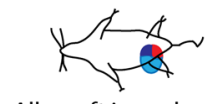

Allograft in nude mice (in vivo screen)

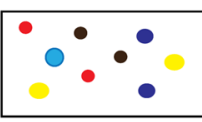

Soft agar assay (in vitro screen)

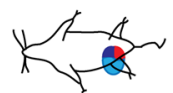

Allograft in nude mice (in vivo screen)

Genomic DNA isolation for TOPO cloning and Sanger sequencing

12 colonies: individually grow in cell culture for 1 week

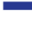

(3 colonies per plates) 25 mice per group;
all have tumors in 30 days

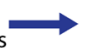

Genomic DNA isolation for targeted PCR and Hi-Seq

Figure 3. Screen with unbiased genome-wide CRISPR sgRNA knockout library without $\operatorname{Tr} p 53$ sgRNAs in MEF-LoxP-KC cells. (A) Schematic of screen with genome-wide CRISPR sgRNA library without Trp53 sgRNAs. (B) Representative colonies were identified in soft agar 3 weeks after transduced MEFs were seeded in soft agar. (C) Representative tumor formation in nude mice allografts after transduced MEFs were intramuscularly injected and followed for 1 month.

Genome-wide CRISPR/Cas9 sgRNA library without Trp53 sgRNAs. Because our previous results demonstrated that Trp53 sgRNAs dominated the soft agar screen, we generated a genome-wide CRISPR/Cas9 knockout sgRNA library without $\operatorname{Trp} 53 \mathrm{sgRNAs}^{18}$. This sgRNA library is comprised of sequences from the mouse Brie library from Doench JG et al., not including Trp53 sgRNAs, and also contains additional sgRNAs targeting microRNAs from the mGeCKO knockout sgRNA library ${ }^{16,17}$. Deep sequencing in Fig. S1 demonstrated good representation of sgRNAs in the library. Then, using this library we performed a second screen in low passage MEF-LoxP-KC cells. After stable transduction with lentivirus containing sgRNAs at 0.2-0.4 MOI, MEFs were plated in soft agar or allografted into nude mice to screen for transformed cells. We performed two independent screens. After transduced MEFs were cultured in soft agar for 3 weeks, the colonies were counted. There were 511 colonies in the first screen group, and 512 colonies in the second screen group (Fig. 3A). The individual colonies were each isolated and grown in cell culture for 1 week after which genomic DNA was isolated by TOPO cloning (Fig. 3B). Three clones from each colony were randomly picked for Sanger sequencing to identify integrated sgRNAs and an additional 7 clones were randomly picked for Sanger sequencing if more than one sgRNA was identified in the first 3 clones. Furthermore, each pool of MEFs transduced with the library was injected into the muscle of 25 nude mice with 2 million cells per mouse. After 30 days, all of the mice developed tumors at the injection site (Fig. 3C). Deep sequencing was performed to identify integrated sgRNAs from genomic DNA isolated from the tumors and compared to sgRNAs of the transduced MEFs before screening in soft agar or in nude mice.

Screen with genome-wide CRISPR/Cas9 library without Trp53 identifies multiple candidate genes that suppress transformation in vitro. A total of 223 out of 511 colonies isolated from the first soft agar screen were able to grow in cell culture. Among these 223 colonies, Sanger sequencing from 216 of these cultured colonies identified only one sgRNA from each colony targeting 7 different genes, including $p 19^{A r f}$ encoded by $C d k n 2 a$ (Fig. 4A). Sanger sequencing of the remaining 7 cultured colonies identified two sgRNAs targeting $C d k n 2 b$ and Mat $2 b$. In the second soft agar screen, a total 230 of 512 isolated colonies grew in cell culture. 229 of 230 cultured colonies contained only one sgRNA and Sanger sequencing results determined that a total of 19 individual sgRNAs targeting 19 different genes were identified (Fig. 4A). p19 ${ }^{\text {Arf }}$ was not identified in this second screen (Fig. 4A). Sanger sequencing of the remaining colony identified two sgRNAs targeting $C d k n 2 b$ and $M a t 2 b$ (Fig. 4A). Interestingly, none of the genes that were identified from colonies harboring a single targeting sgRNA were found in both soft agar screens, but colonies containing sgRNAs targeting both C $d k n 2 b$ and Mat $2 b$ were found in each of the soft agar screens.

Furthermore, deep sequencing was performed to identify the sgRNAs that were integrated into the genome of 50 tumors from the nude mice allograft screen (Fig. 4B). Thirteen sgRNAs were identified in the soft agar screen that were also found in the nude mice allograft screen. However, several sgRNAs were only identified in the nude mice allograft screen, including sgRNAs targeting Mup14, Zbtb14, Artn, Atm, Fbxw7 and Slc9a3. Interestingly, $F B X W 7$ and $S L C 9 A 3$, including several other candidates from the screen in nude mice, were identified as being prone to co-mutation with KRAS in the TCGA data set of all human cancers (Table S1). Overall, we identified several novel candidate genes whose mutation may result in transformation of $\mathrm{Kras}^{\mathrm{G} 12 \mathrm{D}}$ immortalized MEF-LoxP-KC.

Mutation of Slc9a3 transforms Kras $^{\mathrm{G} 12 \mathrm{D}}$ immortalized MEF-LoxP-KC in vitro. To further test the tumor suppressor function of the identified candidate genes, we transduced MEF-LoxP-KC cells with a lentivirus expressing each identified sgRNA targeting individual candidate genes identified from those screens (Table 1). 
A

\begin{tabular}{|c|c|c|}
\hline Gene & Rep-1 counts (223) & Rep- 1 counts (230) \\
\hline Man2b1 & 177 & \\
\hline Mat2b & 20 & \\
\hline$C d k n 2 b$ & 14 & \\
\hline Cdkn2a (p19arf) & 2 & \\
\hline $1 d h 3 g$ & 1 & \\
\hline Lrrc8c & 1 & \\
\hline Fbxw7 & 1 & \\
\hline$C d k n 2 b+M a t 2 b$ & 7 & 1 \\
\hline Fam21 & & 40 \\
\hline 4930452B06Rik & & 112 \\
\hline Ccdc18 & & 50 \\
\hline Nfkbil1 & & 5 \\
\hline Sema4d & & 4 \\
\hline Pax1 & & 2 \\
\hline Ctsz & & 2 \\
\hline Obs/1 & & 2 \\
\hline mmu-mir-7024 & & 2 \\
\hline Slc8a3 & & 1 \\
\hline Gm20865 & & 1 \\
\hline $\mathrm{Hn} 1 \mathrm{I}$ & & 1 \\
\hline$C d k / 3$ & & 1 \\
\hline Filip1 & & 1 \\
\hline Adamts8 & & 1 \\
\hline Lrrc15 & & 1 \\
\hline $\mathrm{Ngfr}$ & & 1 \\
\hline Gabra4 & & 1 \\
\hline 4930407l10Rik & & 1 \\
\hline
\end{tabular}

B

\begin{tabular}{|c|c|c|c|c|c|}
\hline Gene & $\begin{array}{c}\text { Shown } \\
\text { in soft agar }\end{array}$ & $\begin{array}{c}\text { Rep-1 } \\
\text { before screen }\end{array}$ & $\begin{array}{c}\text { Rep-2 } \\
\text { before screen }\end{array}$ & Rep-1 counts & Rep-1 counts \\
\hline Man2b1 & Y & 573 & 15 & 25845831 & 4050 \\
\hline Mat2b & Y & 155 & 12 & 2861864 & 253 \\
\hline Cdkn2b & Y & 139 & 19 & 2141416 & 9990 \\
\hline Fbxw7 & Y & 22 & 20 & 766 & 2727 \\
\hline Mup14 & N & 43 & 12 & 19 & 89471 \\
\hline Fam21 & Y & 17 & 43 & 1248 & 3222407 \\
\hline 4930452B06Rik & Y & 26 & 627 & 4106 & 21888549 \\
\hline Ccdc18 & Y & 0 & 223 & 479 & 4491484 \\
\hline Nfkbil1 & Y & 0 & 10 & 110 & 738387 \\
\hline Zbtb14 & $\mathrm{N}$ & 17 & 8 & 58 & 68563 \\
\hline Artn & $\mathrm{N}$ & 13 & 8 & 54802 & 1 \\
\hline Lrrc8c & $\mathrm{Y}$ & 8 & 0 & 35656 & 11 \\
\hline Filip1 & $\mathrm{Y}$ & 2 & 7 & 12909 & 2705 \\
\hline Sema4d & $\mathrm{Y}$ & 11 & 19 & 1 & 2033 \\
\hline Fbxo42 & $\mathrm{Y}$ & 17 & 2 & 354 & 71 \\
\hline Obs/1 & $\mathrm{Y}$ & 0 & 9 & 5 & 13 \\
\hline Atm & $\mathrm{N}$ & 52 & 28 & 2 & 11 \\
\hline SIc9a3 & $\mathrm{N}$ & 15 & 21 & 2 & 4 \\
\hline Cdkn2a (p19Arf) & $\mathrm{Y}$ & 27 & 11 & 9 & 5 \\
\hline
\end{tabular}

Figure 4. List of candidate genes that suppress transformation from screen with genome-wide CRISPR sgRNA library without $\operatorname{Trp} 53$ sgRNAs. List of candidate genes identified by (A) the soft agar assay and from (B) nude mice allografts.

\begin{tabular}{|l|}
\hline Target gene \\
\hline Kansl2 \\
\hline Izumo2 \\
\hline Slmap \\
\hline Fbxw7 \\
\hline Filip1 \\
\hline Fbxo42 \\
\hline Man2b1 \\
\hline Cdkn2b \\
\hline Mat2b \\
\hline Fam21 \\
\hline $4930452 B 06$ Rik \\
\hline Obsl1 \\
\hline Sema4d \\
\hline Ccdc18 \\
\hline Nfkbil1 \\
\hline Zbtb14 \\
\hline Mup14 \\
\hline Artn \\
\hline Lrrc8c \\
\hline Atm \\
\hline Slc9a3 \\
\hline
\end{tabular}

Table 1. The list of 21 candidate genes.

Then, transduced cells were plated in soft agar and allografted into nude mice. Colony formation in soft agar and tumor growth in nude mice allografts were only detected in MEF-LoxP-KC cells transduced with lentivirus expressing sgRNA targeting either $p 19^{A r f}, F b x w 7$ or $S l c 9 a 3$ (Fig. 5A). To further confirm the tumor suppression function of $F b x w 7$ and $S l c 9 a 3$, we transduced MEF-LoxP-KC cells with a different sgRNA targeting either $F b x w 7$ or Slc9a3. While MEF-LoxP-KC cells transduced with a different sgRNA targeting Slc9a3 consistently formed colonies in soft agar and tumors in nude mice allografts (Fig. 5B), we did not detect discernable colony formation 


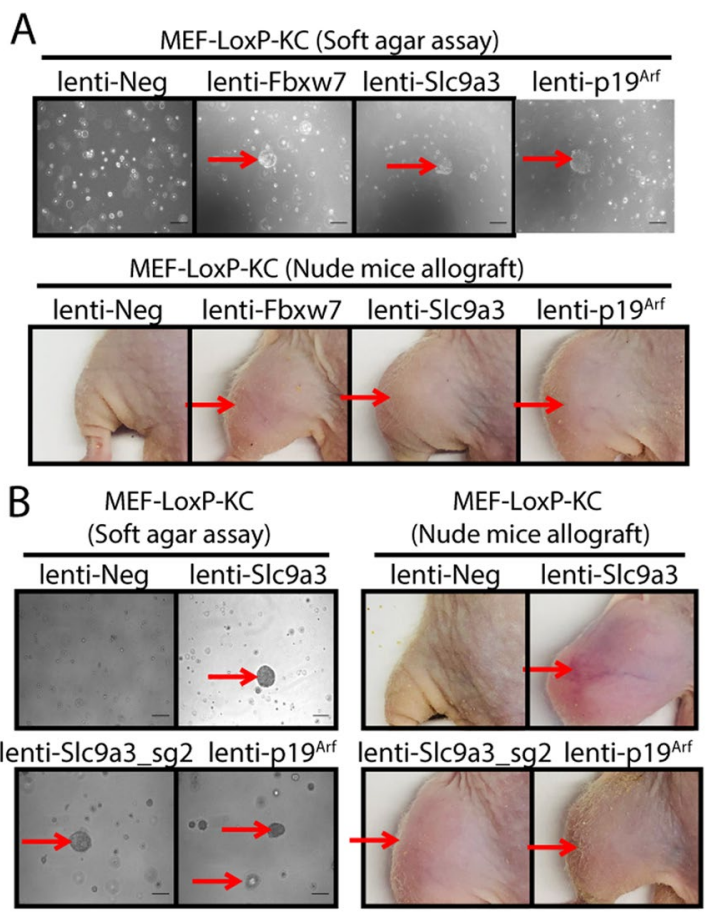

Figure 5. Individual validation of candidate genes that suppress transformation from the screen with genomewide CRISPR sgRNA library without Trp53 sgRNAs. (A) MEF-LoxP-KC cells transduced with a single candidate sgRNA were plated in soft agar or injected into the muscle of nude mice $(n=2$ or 3$)$. (B) MEFLoxP-KC cells transduced with more than one sgRNA targeting Slc9a3 were plated in soft agar assay or injected into the muscle of nude mice ( $n=2$ or 3 ). The result is representative of at least two independent experiments. Scale bars $=100 \mu \mathrm{m}$.

in MEF-LoxP-KC cells transduced with a different sgRNA targeting Fbxw7. Thus, our results identify $S l c 9 a 3$ as a potential tumor suppressor whose mutation in $\mathrm{Kras}^{\mathrm{G} 12 \mathrm{D}}$-expressing MEFs induces transformation.

Expression of endogenous $\operatorname{Kras}^{\mathrm{G} 12 \mathrm{D}}$ and CRISPR/Cas9 in vivo editing of either Trp53 or $p 19^{A r f}$, but not Slc9a3, induces primary sarcomas. Because mutation of $\operatorname{Tr} p 53$ or $C d k n 2 a$ is sufficient to initiate sarcomagenesis with expression of endogenous $\mathrm{Kras}^{\mathrm{G} 12 \mathrm{D} 19}$, we next investigated whether mutation of the identified candidate genes could induce primary sarcomas in $\mathrm{Kras}^{\mathrm{LSL}-\mathrm{G} 12 \mathrm{D} /+} ;$ Rosa26 $^{\mathrm{LoxP}-\mathrm{Cas} 9-\mathrm{EGFP} /+}$ (K-LoxP-C) mice. We applied our recently reported in vivo electroporation method ${ }^{15}$ to intramuscularly deliver a naked plasmid px333-Cre-sgRNA that contains a sgRNA targeting only $S l c 9 a 3$ or a mixed pool of px333-Cre-sgRNAs that contain sgRNAs targeting the other 20 candidate genes that are from the screens (Table 1) either including $p 19^{A r f}$ or excluding $p 19^{A r f}$. In addition, K-LoxP-C mice that underwent in vivo electroporation with a non-targeting sgRNA served as a negative control and $\mathrm{K}$-LoxP-C mice that underwent in vivo electroporated with a sgRNA targeting either Trp53 alone or $p 19^{A r f}$ alone served as positive controls. All mice were followed for up to 1 year after injection. Interestingly, we only detected primary tumors in the positive control groups and the group of mice injected with the mixed pool of px333-Cre-sgRNAs including the p19Arf sgRNA (Fig. 6). We examined the efficiency of CRISPR/Cas9 editing in primary tumors generated by the mixed pool of sgRNAs including the $p 19^{A r f}$ sgRNA, and we detected indels in multiple genes, including $p 19^{A r f}$ and $F b x w 7$. In addition, the primary tumors initiated by a sgRNA targeting Trp53 or $p 19^{\text {Arf }}$ or the mixed pool of sgRNAs including the $p 19^{A r f}$ sgRNA have similar histologies on haematoxylin and eosin-stained sections (Fig. S4). Taken together, these data suggest that we achieved efficient CRISPR/Cas9 editing in vivo and that the mutation of $p 19^{A r f}$ was required to generate primary sarcomas (Fig. S2). Overall, our CRISPR/Cas9 in vivo editing results suggest that mutation of either Trp53 or $p 19^{A r f}$ is necessary to cooperate with $\mathrm{Kras}^{\mathrm{G} 12 \mathrm{D}}$ to induce primary sarcomas.

\section{Discussion}

We systematically searched for genes whose mutation together with expression of oncogenic Kras could transform MEFs. By using low passage Kras ${ }^{\mathrm{G} 12 \mathrm{D}}$ immortalized MEFs that constitutively express Cas9 from the Rosa26 locus, we were able to efficiently transduce mouse genome-wide CRISPR/Cas9 knockout sgRNA libraries to perform a screen in cells with an identical genetic background. Because the Trp53 sgRNAs dominated the first unbiased genome-wide CRISPR/Cas9 knockout screen, which limited the detection of other sgRNAs, we performed a second screen using a mouse genome-wide sgRNA library excluding Trp53 sgRNAs. We identified several novel candidate genes using this library. Therefore, this genome-wide sgRNA library that excludes Trp53 sgRNAs may be useful for other screens to search for tumor suppressor genes. For instance, this library could be potentially 


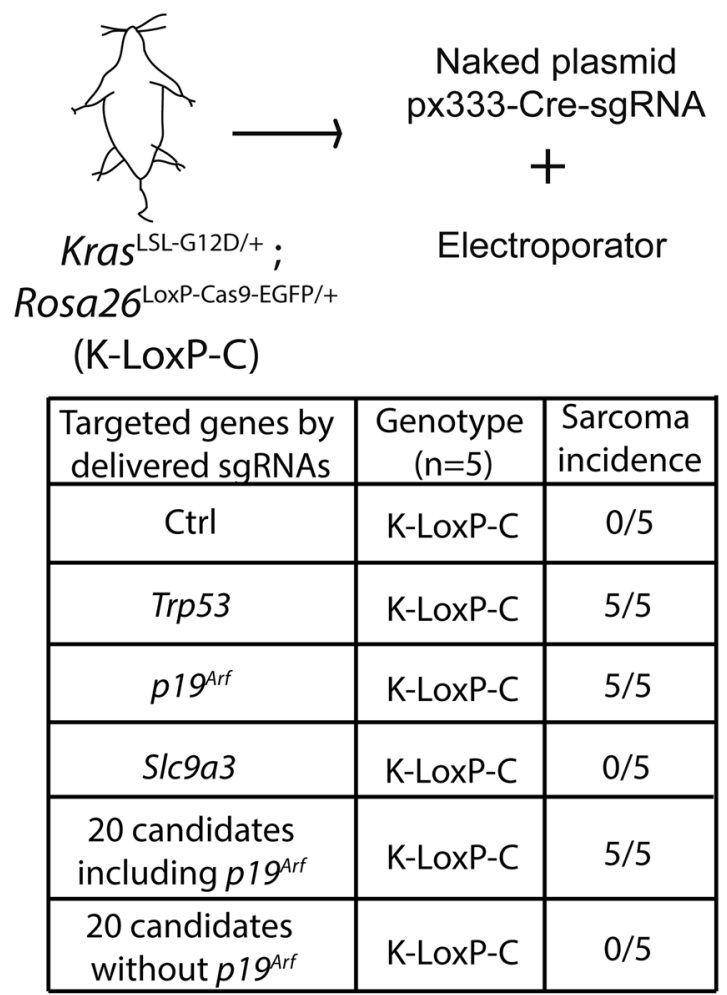

In vivo electroporation

Figure 6. Testing candidate genes in vivo. Naked plasmid px333-Cre-sgRNAs containing different sgRNAs were electroporated in vivo into K-LoxP-C mice and the mice were followed up to 1 year for sarcoma formation $(n=5)$.

applied to low passage epithelial cells isolated from the K-LoxP-C mice to identify tumor suppressor genes in cell types other than MEFs.

In addition to the well-described tumor suppressor $C d k n 2 a$, our screen identified several candidate genes such as $\mathrm{Fbxw7}$ and $S l c 9 a 3$, which may cooperate with oncogenic Kras ${ }^{\mathrm{G} 12 \mathrm{D}}$ to transform cells. Tumor suppressor FBXW7, a component of the Skp1-Cullin1-Fbox E3 ubiquitin ligase complex, is frequently mutated in several types of human cancers ${ }^{20}$. Loss of $F B X W 7$ increases the expression of its oncogenic substrates, such as CCNE, MYC, NOTCH1, and JUN, which contribute to tumorigenesis ${ }^{21}$. Although we observed transformation of Cas9 expressing $\mathrm{Kras}^{\mathrm{G} 12 \mathrm{D}}$ immortalized MEFs with one sgRNA to Fbxw7, we did not observe transformation with a second sgRNA to Fbxw7 (Fig. S3). Moreover, we failed to initiate sarcomas in K-LoxP-C mice $(\mathrm{n}=5)$ electroporated with a sgRNA to $F b x w 7$. Therefore, it is conceivable that the single active $F b x w 7$ sgRNA is driving transformation by mutating an off-target site in the genome. However, conditional deletion of Fbxw7 accelerates Kras ${ }^{\mathrm{G} 12 \mathrm{D}}$ driven pancreatic cancer in a genetically engineered mouse model ${ }^{21}$. Moreover, human cancers in the TCGA database are prone to co-mutate KRAS and FBXW7 (Table S1). Therefore, we cannot exclude the possibility that $F b x w 7$ is a weak tumor suppressor, which would be sufficient to drive tumorigenesis with Kras mutation in a small number of mice if we utilized a much larger cohort of mice for the in vivo electroporation experiment. In addition, it is possible that co-mutation of Kras with certain Fbxw7 mutations transforms cells and drives cancer development in vivo in a cell-type dependent manner.

SLC9A3 is also known as sodium-hydrogen exchanger 3 (NHE3). The SLC9 family of proteins play a critical role in electroneutral exchange of $\mathrm{Na}^{+}$and $\mathrm{H}^{+}$in mammalian cells and also regulate cell proliferation, apoptosis, and cell migration ${ }^{22}$. We found that two independent sgRNAs to Slc9a3 transformed Cas9 expressing Kras ${ }^{\text {G12D }}$ immortalized MEFs. Moreover, SLC9A3 is found co-mutated with KRAS in human cancers in the TCGA database

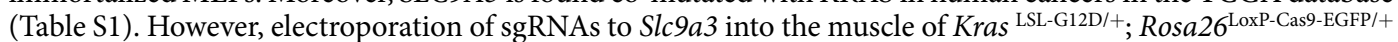
$(\mathrm{K}-$ LoxP-C) mice $(\mathrm{n}=5)$ failed to initiate sarcomagenesis. Similarly, with a sample size of 5 mice, we cannot exclude the possibility that $S l c 9 a 3$ is a weak tumor suppressor in the in vivo electroporation assay that may be sufficient to drive tumorigenesis. Taken together, these results suggest that mutation of Kras and Slc9a3 may transform cells and drive tumorigenesis in a cell-type dependent manner. Therefore, in future studies, it would be interesting to test in mice if mutations in Kras and $S l c 9 a 3$ promote cancer development in tissues where these genes are frequently found co-mutated in human cancers (Table S1). Furthermore, FBXW7 is significantly co-mutated with KRAS in colon cancer and ATM is significantly co-mutated with KRAS in pancreatic cancer (Table S4). Therefore, our list of candidate genes could be potentially validated in other types of cancer models.

Compared to sophisticated in vivo immunocompetent models, in vitro soft agar assays and in vivo cellular transplantation in immunocompromised mice have advantages for performing high throughput testing of a large number of candidate genes in a relatively short time. Therefore, in vitro soft agar assays and in vivo cellular 
transplantation in immunocompromised mice are valuable tools to identify candidate genes associated with tumorigenesis. However, our in vivo studies indicate that the results from the in vitro and transplant models may not be recapitulated in immunocompetent autochthonous models. Unfortunately, in many studies, including this one, mutating the candidate genes in vivo within a native tissue microenvironment fails to initiate cancer ${ }^{23,24}$. This discordance may simply reflect differences in the requirements between transformation of MEFs and in vivo sarcomagenesis. For example, it is possible that in vivo the immune system or other cells in the microenvironment play critical roles to prevent cancer initiation and progression ${ }^{25}$. One potential limitation of our in vitro CRISPR/Cas9 screen is that candidate genes that were mutated in immortalized MEFs may have created a growth disadvantage in vitro that would not occur in vivo. To address this limitation, in future experiments whole genome screening could be performed in vivo in the absence of in vitro culture. However, it is also possible that the candidate genes have the potential to promote tumorigenesis in vivo when mutations in these genes occur within a permissive cell type and developmental stage.

Our unbiased genome-wide approach using a CRISPR/Cas9 knockout screen for transformation in ras $^{\mathrm{G} 12 \mathrm{D}}$ immortalized MEFs successfully identified well-known tumor suppressors Trp53 and $p 19^{A r f}$ and also candidate genes, such as $\mathrm{Fbxw} 7$ and $\operatorname{Slc9a3}$. We tested the potential tumor suppressor function of these genes in vivo using our recently reported CRISPR/Cas9 initiated $K r a s^{\mathrm{G} 12 \mathrm{D}}$-driven sarcoma model ${ }^{15}$. However, only mutation of $\operatorname{Tr} p 53$ or its upstream regulatory gene $p 19^{A r f}$ could cooperate with $\mathrm{Kras}^{\mathrm{G} 12 \mathrm{D}}$ to initiate primary sarcomas in skeletal muscle of adult mice. Therefore, the p $19^{\text {Arf }}$-p53 pathway appears to be the critical tumor suppressor pathway to cooperate with oncogenic Kras to lead to in vivo sarcoma development. Because no Trp53 target genes were identified in this screen, our results are consistent with a model where multiple $\operatorname{Tr} p 53$ downstream genes and pathways function simultaneously to suppress transformation ${ }^{26}$. To search for genes in addition to the p19 ${ }^{\text {Arf }}$-p53 pathway that can promote sarcoma development, in the future we plan to perform an in vivo CRISPR/Cas9 screen in immune competent mice for genes that can cooperate with $\operatorname{Tr} 553$ mutation. This approach has proven to be a useful tool to identify novel genes associated with tumorigenesis in mouse models of glioblastoma ${ }^{27}$, liver can$\operatorname{cer}^{28}$, and lung cancer ${ }^{29}$.

In conclusion, we identified $\mathrm{Fbxw} 7$ and $\operatorname{Slc} 9 a 3$ as genes that when mutated can transform $\mathrm{Kras}{ }^{\mathrm{G} 12 \mathrm{D}}$ immortalized MEFs, but are unable to cooperate with oncogenic Kras to drive sarcomagenesis in the muscle of adult mice in vivo. Our mouse genome-wide sgRNA library excluding Trp53 sgRNAs may be a useful tool for other researchers who perform screens where the dominant sgRNAs selected target Trp53.

\section{Methods}

Mice. $\quad \mathrm{Kras}^{\mathrm{LSL}-\mathrm{G} 12 \mathrm{D} /+}$ mice $^{30}$ were a gift from T. Jacks (Massachusetts Institute of Technology). Rosa26 $6^{\mathrm{LSL}-\mathrm{Cas} 9-\mathrm{EGFP} /+}$ mice ${ }^{31}$ were obtained from Jackson labs. Rosa $26^{\text {LoxP-Cas9-EGFP/+ }}$ mice were generated by methods described previously ${ }^{15}$. Athymic nude (nu/nu) mice (5 to 6 weeks old) for the allograft study were purchased from Taconic Biosciences and maintained in Duke University's accredited animal facility. MEFs were transduced with lentivirus and 2 million cells were injected into the hind limbs of nude mice. All animal studies were performed in accordance with protocols approved by the Duke University Institutional Animal Care and Use Committee (IACUC).

MEF isolation and transduction with adeno-cre. E13.5 to E14.5 embryos isolated from pregnant mice were used to isolate MEFs using a standard protocol. To activate expression of Cas9 from the Rosa26 locus and to express Kras ${ }^{\mathrm{G} 12 \mathrm{D}}$ from the endogenous Kras locus, MEFs were infected with an adenovirus expressing Cre recombinase (University of Iowa).

Molecular analysis of recombination. Recombination of the Cas9 allele and mutant Kras allele were assessed by PCR using methods described previously ${ }^{15}$ with primers in Supplementary Tables 2 and 3.

Histology, immunohistochemistry, and tumor analysis. Tissues were harvested and fixed in $4 \%$ formalin and paraffin-embedded. Hematoxylin and eosin staining was performed using standard methods.

Genomic DNA isolation, PCR amplification and indel analysis. Cells and tissues were harvested for genomic DNA isolation using DNeasy Blood \& Tissue Kit (Qiagen, 69504). PCR amplification, re-annealing process and CRISPR/Cas9 induced indels on different targeted genes were performed using methods described previously ${ }^{15}$. PCR amplification of targeted genes was subjected to a re-annealing process to enable heteroduplex formation and indels were further detected using the Surveyor Mutation Detection Kit (IDT, 706021) following the standard protocol.

CRISPR screens. Mouse Gecko v2 CRISPR libraries in the lentiguide-Puro vector were provided by Feng Zhang (MIT), made available through Addgene. A mouse genome-scale library without Trp53 sgRNAs was created with sgRNA sequences targeting coding genes and non-targeting controls taken from the Brie library and sgRNA sequences targeting miRNAs taken from the Gecko library for a total of 84,077 $\operatorname{sgRNAs}{ }^{16,18,32}$. Oligonucleotide pools were ordered from Twist Bioscience and cloned into the lentiguide-Puro vector by Gibson assembly (NEB). Transformations of the assembled library were done into Endura competent cells (Lucigen) to provide 66-fold coverage of the library and sgRNA representation was verified by high-throughput sequencing. Lentivirus for the CRISPR libraries was prepared and MEFs were transduced under conditions to achieve MOI of $0.2-0.4$ as described ${ }^{33}$. Cells were selected with puromycin for 48 hours and expanded for one week in vitro before plating into soft agar or injection into nude mice.

Deep sequencing. Genomic DNA from cells or tumors was prepared by QiaAmp Blood Maxi Kit according to the manufacturer's instructions. 
The sgRNAs from each sample were PCR amplified using primers with Illumina adaptors and barcodes for multiplexing and sequenced on the Illumina HiSeq. 2500. Reads were trimmed and aligned to the library reference to generate read count files for each sgRNA using MAGeCK ${ }^{34}$.

In vivo electroporation. All in vivo electroporations were performed using methods described previously ${ }^{15}$. $50 \mu \mathrm{l}$ of $1 \mu \mathrm{g} / \mathrm{ul}$ naked DNA plasmids diluted in sterile saline was injected into the gastrocnemius using an insulin syringe. Then, a pair of needle electrodes was inserted into the muscle at the site of injection, and electric pulses were delivered using an electric pulse generator (Electro Square Porator ECM830; BTX, San Diego, CA).

Senescence assay. Cells grown in tissue culture plates were subjected to a senescence assay using the manufacturer's standard protocol (Cell Signaling Technology, 9860S).

Soft agar assay. $\quad 1.8 \%$ agar (BD Diagnostics, 204010) was made in de-ionized water and autoclaved. $2 \mathrm{x}$ cell culture medium was made by dissolving 1 bag of High glucose DMEM powder (Thermo Fisher Scientific, 12800017) and $1.85 \mathrm{~g}$ sodium bicarbonate (Thermo Fisher Scientific, 25080-094) in $500 \mathrm{ml}$ of de-ionized water with $\mathrm{pH}$ adjusted to 6.8. The medium was sterilized by passage through a $0.2 \mu \mathrm{m}$ filter. Sterilized medium was mixed with 20\% FBS and 1\% antibiotic-antimycotic (Thermo Fisher Scientific, 10091148). $2.5 \mathrm{ml}$ of $0.6 \%$ agar medium consisting of $1.8 \%$ agar, $2 \mathrm{x}$ cell culture medium, and $1 \mathrm{x}$ cell culture medium as described previously ${ }^{15}$ was poured into 6-well plates. $0.25 \mathrm{ml}$ medium containing 50,000 cells was mixed with $0.5 \mathrm{ml}$ of $0.6 \%$ agar medium and was poured onto the top of the $0.6 \%$ agar medium in the plate. Plates were incubated at $37^{\circ} \mathrm{C}$ with $5 \% \mathrm{CO}_{2}$ in a humidified cell-culture incubator and were fed with $0.5 \mathrm{ml}$ of $1 \mathrm{x}$ cell culture medium every week. Colonies in the soft agar assay were counted, imaged, and picked for re-growth in tissue culture for 3 to 4 weeks.

Co-mutation analysis. To analyze which mutations were enriched in Kras mutant cancers, we accessed the AACR GENIE dataset via cbioportal (http://genie.cbioportal.org/login.jsp). We then selected the "GENIE Cohort v6.1-public". Then we used the "enhanced study view" feature and selected either "Colorectal Cancer", "Non-Small Cell Lung Cancer", "Sarcoma" or "Pancreatic Cancer". We recorded the case ID's associated with these cancer types, then returned to the home page (http://genie.cbioportal.org/) and chose "Query by Gene", then input our the case ID's of one of the cancers. Then, selecting for only Somatic Mutation, we queried for "KRAS". On the following screen we then selected "enrichments" and chose the "mutation tab", and selected the "Co-Occurrence" option on the screen. We then were able to sort by q-value and identify the top ten mutations with the highest tendency for co-occurrence for that cancer. We repeated these steps for each cancer type, and also for all cancer types combined.

Received: 7 July 2019; Accepted: 28 October 2019;

Published online: 20 November 2019

\section{References}

1. Hanahan, D. \& Weinberg, R. A. Hallmarks of cancer: the next generation. Cell 144, 646-74 (2011).

2. Burmer, G. C. \& Loeb, L. A. Mutations in the KRAS2 oncogene during progressive stages of human colon carcinoma. Proc Natl Acad Sci USA 86, 2403-7 (1989).

3. Almoguera, C. et al. Most human carcinomas of the exocrine pancreas contain mutant c-K-ras genes. Cell 53, 549-54 (1988)

4. Tam, I. Y. S. et al. Distinct epidermal growth factor receptor and KRAS mutation patterns in non-small cell lung cancer patients with different tobacco exposure and clinicopathologic features. Clin Cancer Res 12, 1647-53 (2006).

5. Hill, M. A. et al. Detection of K-ras mutations in resected primary leiomyosarcoma. Cancer Epidemiol Biomarkers Prev 6, 1095-100 (1997).

6. Scheffler, M. et al. K-ras Mutation Subtypes in NSCLC and Associated Co-occuring Mutations in Other Oncogenic Pathways. J Thorac Oncol 14, 606-16 (2019).

7. Arbour, K. C. et al. Effects of Co-occurring Genomic Alterations on Outcomes in Patients with KRAS-Mutant Non-Small Cell Lung Cancer. Clin Cancer Res 24, 334-40 (2018)

8. Tuveson, D. A. et al. Endogenous oncogenic K-rasG12D stimulates proliferation and widespread neoplastic and developmental defects. Cancer Cell 5, 375-87 (2004).

9. Brady, C. A. et al. Distinct p53 transcriptional programs dictate acute DNA-damage responses and tumor suppression. Cell 145, 571-83 (2011).

10. Kaiser, A. M. \& Attardi, L. D. Deconstructing networks of p53-mediated tumor suppression in vivo. Cell Death Differ 25, 93-103 (2018).

11. Joung, J. et al. Genome-scale CRISPR-Cas9 knockout and transcriptional activation screening. Nat Protoc 12, 828-63 (2017).

12. Katigbak, A. et al. A CRISPR/Cas9 Functional Screen Identifies Rare Tumor Suppressors. Sci Rep 6, 38968 (2016).

13. Song, C.-Q. et al. Genome-Wide CRISPR Screen Identifies Regulators of Mitogen-Activated Protein Kinase as Suppressors of Liver Tumors in Mice. Gastroenterology 152(1161-73), el (2017).

14. Guo, X. et al. Site-specific proteasome phosphorylation controls cell proliferation and tumorigenesis. Nat Cell Biol 18, 202-12 (2016).

15. Huang, J. et al. Generation and comparison of CRISPR-Cas9 and Cre-mediated genetically engineered mouse models of sarcoma. Nat Commun 8, 15999 (2017).

16. Sanjana, N. E., Shalem, O. \& Zhang, F. Improved vectors and genome-wide libraries for CRISPR screening. Nat Methods 11, 783-4 (2014).

17. Chen, S. et al. Genome-wide CRISPR screen in a mouse model of tumor growth and metastasis. Cell 160, 1246-60 (2015).

18. Doench, J. G. et al. Optimized sgRNA design to maximize activity and minimize off-target effects of CRISPR-Cas9. Nat Biotechnol 34, 184-91. (2016).

19. Kirsch, D. G. et al. A spatially and temporally restricted mouse model of soft tissue sarcoma. Nat Med 13, 992-7 (2007).

20. Akhoondi, S. et al. FBXW7/hCDC4 is a general tumor suppressor in human cancer. Cancer Res 67, 9006-12 (2007).

21. Zhang, Q. et al. Fbxw7 Deletion Accelerates KrasG12D-Driven Pancreatic Tumorigenesis via Yap Accumulation. Neoplasia 18, 666-73. (2016).

22. Xu, H., Ghishan, F. K. \& Kiela, P. R. SLC9 Gene Family: Function, Expression, and Regulation. Compr Physiol 8, 555-83 (2018) 
23. Lin, P. P. et al. Targeted mutation of $\mathrm{p} 53$ and Rb in mesenchymal cells of the limb bud produces sarcomas in mice. Carcinogenesis 30 , $1789-95$ (2009)

24. Nechiporuk, T. et al. The REST remodeling complex protects genomic integrity during embryonic neurogenesis. Elife 5, e09584 (2016).

25. Chen, D. S. \& Mellman, I. Oncology meets immunology: the cancer-immunity cycle. Immunity 39, 1-10 (2013).

26. Kastenhuber, E. R. \& Lowe, S. W. Putting p53 in Context. Cell 170(78), 1062 (2017).

27. Chow, R. D. et al. AAV-mediated direct in vivo CRISPR screen identifies functional suppressors in glioblastoma. Nat Neurosci, https://doi.org/10.1038/nn.4620 (2017).

28. Wang, G. et al. Mapping a functional cancer genome atlas of tumor suppressors in mouse liver using AAV-CRISPR-mediated direct in vivo screening. Sci Adv 4, eaao5508 (2018).

29. Rogers, Z. N. et al. A quantitative and multiplexed approach to uncover the fitness landscape of tumor suppression in vivo. Nat Methods 14, 737-42 (2017).

30. Tuveson, D. A. et al. Endogenous oncogenic K-ras(G12D) stimulates proliferation and widespread neoplastic and developmental defects. Cancer Cell 5, 375-87 (2004).

31. Platt, R. J. et al. CRISPR-Cas9 knockin mice for genome editing and cancer modeling. Cell 159, 440-55 (2014).

32. Joung, J. et al. Genome-scale CRISPR-Cas9 knockout and transcriptional activation screening. Nat Protocols 12, 828 (2017).

33. Joung, J. et al. Genome-scale activation screen identifies a lncRNA locus regulating a gene neighbourhood. Nature 549, 418 (2017).

34. Li, W. et al. MAGeCK enables robust identification of essential genes from genome-scale CRISPR/Cas9 knockout screens. Genome Biol 15, 554 (2014).

\section{Acknowledgements}

We thank Nerissa Williams for care of mice and Andrea Daniel for critically reading this manuscript. This work was supported by the National Cancer Institute of the U.S. NIH under awards R35CA197616 (DGK), T32GM007171 (MC) and F30CA206424 (MC).

\section{Author contributions}

J.H., S.Y.K., D.G.K. designed experiments. J.H., M.C., L.L., E.X., Y.M., W.H. performed experiments. S.Y.K. generated the sgRNA library and provided library lentivirus preparations and processing and analysis for highthroughput sequencing of samples. J.H. and M.C. performed CRISPR screen in soft agar. J.H. performed in vivo CRISPR screen in nude mice. J.H. and E.X. performed in vivo electroporation. T.S.K. and C.A.G. helped to design the sgRNA library without Trp53 sgRNAs. L.L. performed genotyping PCR. Y.M. performed histology. D.M.C. reviewed histology. W.F. analyzed genes co-mutated with KRAS in 4 human cancer types. J.H. and D.G.K. drafted the original manuscript and all authors edited the manuscript.

\section{Competing interests}

D.G.K. is a co-founder of XRAD Therapeutics, which is developing radiosensitizers, and D.G.K. is on the scientific advisory board and owns stock in Lumicell Inc, which is developing intraoperative imaging, but these interests do not overlap with the work in this manuscript.

\section{Additional information}

Supplementary information is available for this paper at https://doi.org/10.1038/s41598-019-53572-w.

Correspondence and requests for materials should be addressed to D.G.K.

Reprints and permissions information is available at www.nature.com/reprints.

Publisher's note Springer Nature remains neutral with regard to jurisdictional claims in published maps and institutional affiliations.

Open Access This article is licensed under a Creative Commons Attribution 4.0 International License, which permits use, sharing, adaptation, distribution and reproduction in any medium or format, as long as you give appropriate credit to the original author(s) and the source, provide a link to the Creative Commons license, and indicate if changes were made. The images or other third party material in this article are included in the article's Creative Commons license, unless indicated otherwise in a credit line to the material. If material is not included in the article's Creative Commons license and your intended use is not permitted by statutory regulation or exceeds the permitted use, you will need to obtain permission directly from the copyright holder. To view a copy of this license, visit http://creativecommons.org/licenses/by/4.0/.

(C) The Author(s) 2019 